\title{
Targets Classification on Multispectral Images using Connectionists Methods
}

\author{
Samir Zeriouh, Mustapha Boutahri, Said El Yamani, Ahmed Roukhe \\ Information Optronic Treatment Team, Atomic, Mechanical, Photonic and Energy Laboratory, Faculty of Science, Moulay Ismail University, \\ Zitoune, Meknès, Morocco
}

Email address:

szeriouh@live.fr (S. Zeriouh)

\section{To cite this article:}

Samir Zeriouh, Mustapha Boutahri, Said El Yamani, Ahmed Roukhe. Targets Classification on Multispectral Images using Connectionists Methods. American Journal of Physics and Applications. Vol. 3, No. 3, 2015, pp. 86-91. doi: 10.11648/j.ajpa.20150303.14

\begin{abstract}
Identification of targets on remote sensing images depends mainly on the databases representing different classes. In this context, this paper proposes a connectionist system using a two-dimensional Kohonen self-organizing map to build a database of some identified targets on a multi-band satellite image. After an enhancing process, essentially based on a non-linear filtering, the system performs a non-supervised classification of a reference image in order to extract the sequence of samples of the reflectance coefficients related to the desired targets. This classification will be used later for automatic extraction of these targets on other stages.
\end{abstract}

Keywords: Multispectral Image, Classification, Identification, Artificial Neural Networks, Self-Organizing Map

\section{Introduction}

The use of topographic maps remains of paramount importance in several areas such as agriculture, hydrography and geography. Their usefulness is also necessary and of high importance in the military field. Indeed, the movement of ground vehicles and gear in unknown terrain, especially in war, is generally based on conventional techniques of topographic maps readings, which provide a global view of the field to cross (Fig. 1). However these maps do not take into account the nature of soil and all the changes that can occur on the maneuver field due to climate and human factors (moist, flooded areas, wetlands and woodlands.). The topographic data become less reliable and could therefore affect the progress of the mobility means on the battlefield.

As part of the defense sector (military intelligence), our job is specifically to classify areas with barriers to mobility, such as swamps, dense forests and surface water, on a reference portion of land. This will be used for detection and subsequent identification on satellite images of other regions.

On a multispectral image of the Kenitra region (Gharb), and after applying a nonlinear median filter type for the purpose of improvement, we operated a non-supervised Artificial Neural Networks (ANNs) classification. Subsequently, by comparing the data to the terrain, we extract the spectral signatures of different regions and points representing the existence of forests, surface water or wetlands. This allowed us to build diverse databases of different aspects of interest.

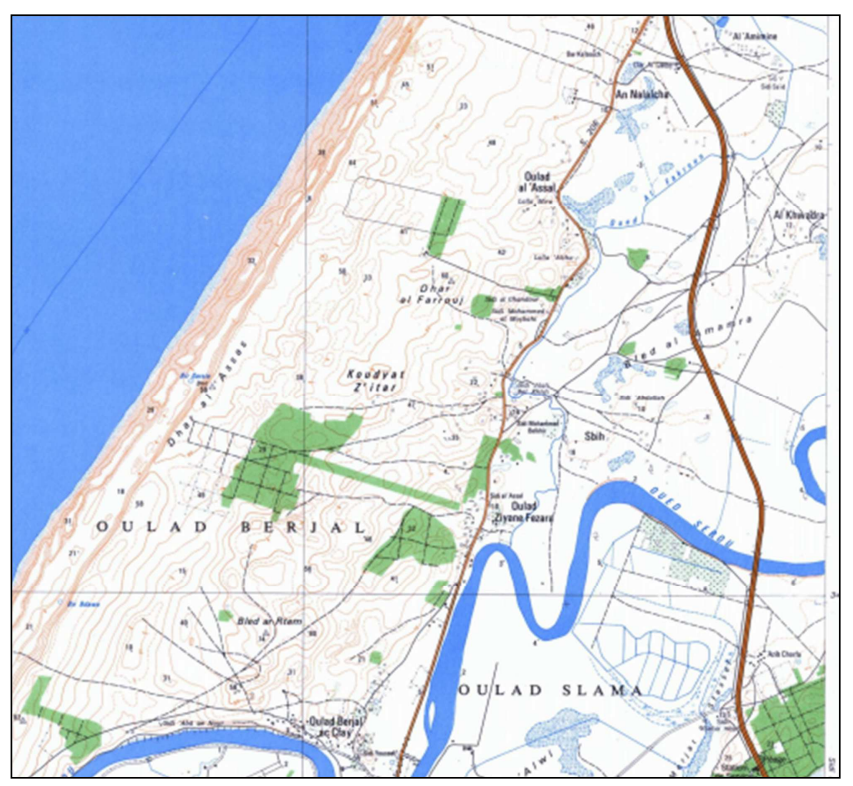

Figure 1. Topographic map of Kenitra region, scale: 1/50 000.

\section{Preprocessing of the Reference Image}

To build our database, we used a satellite image of eight 
bands of Kenitra region, taken by an ETM +1 sensor on board the Landsat-7 satellite on February 25th, 2011. We excluded the panchromatic band (Number. 8) since it has a wavelength bands covering numbers 2, 3 and 4 (green, blue and near-infrared). A colored composition of three strips $(393 \times 473$ window) of the aforesaid image is shown in Figure .2 .

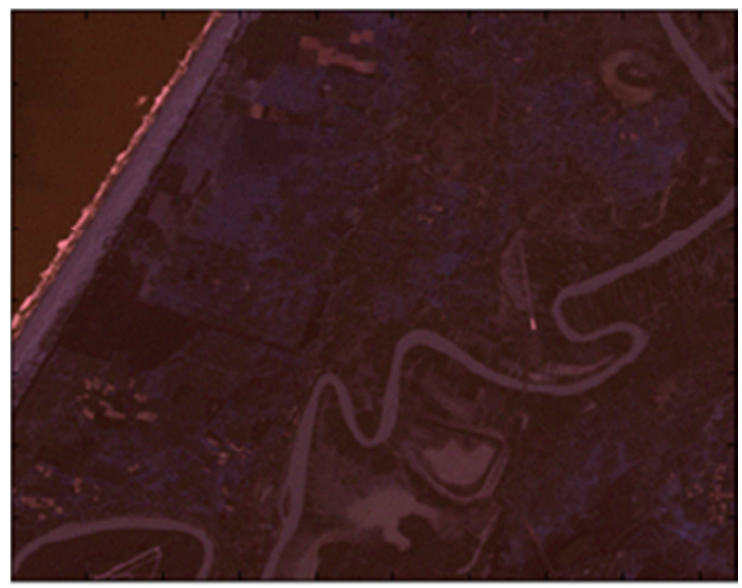

(a)

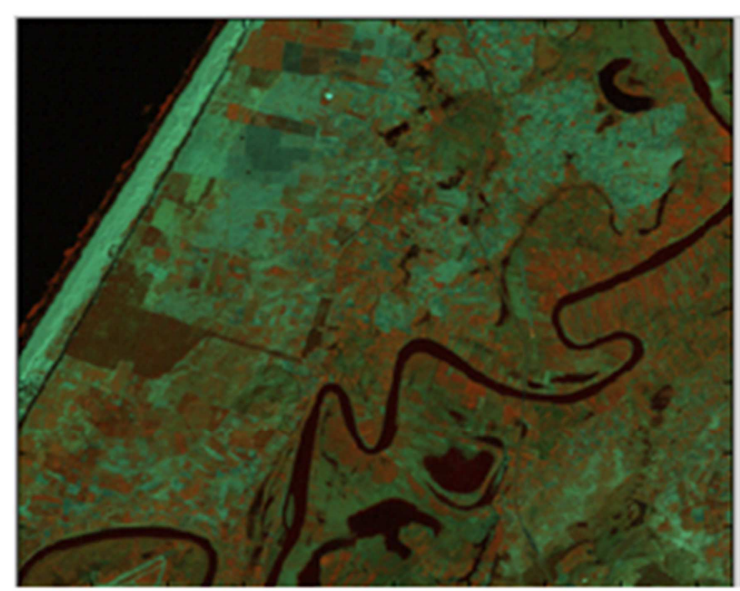

(b)

Figure 2. Colored composition of three Landsat 7 bands of Kenitra region (aVisible spectrum: combination 1, 2 and 3. b-Infrared domain: combination $4,5$ and 7$)$

The choice of this region is based on the fact that it encompasses wetlands, a river, wooded plots of land and part of the sea. This aspect allows for consistent classes matching our targets and therefore facilitates their extraction.

Upon acquisition of such images, a quality degradation is often observed due to some factors usually related to interactions between the acquisition system, air and soil

1 High resolution multispectral scanning Radiometer providing radiation in the visible and infrared eight distinct bands. It is an improved version of the sensor Thematic Mapper (TM), which provides increased spatial resolution thermal infrared band (band 6), improved radiometric calibration and addition of a panchromatic band. It is designed to collect and detect the radiation of earth in a $185 \mathrm{~km}$ band wide. behavior [1]. In our study, and for effective distinction of classes, we are interested in the speckle due to random interference between the waves at the surface reflection [2]. The origin of this noise (salt and pepper) is in the infinity of elementary reflectors composing a resolution cell. He then takes the form of a multiplicative noise where the intensity measured in the respective resolution cell $(i, j)$ is the product of radar reflectivity and a noise-related term $s$.

$$
I(i, j)=r(i, j) * s(i, j)
$$

In order to eliminate these cumulative noise on different bands, we conducted a non-linear filtering of the seven bands, with a median filter [3]. This allowed us not only to attenuate the high frequencies (isolated pixels), but also reduce the uniform or Gaussian additive noise while preserving edges (Fig. 2) [4].

The principle of this filter is to move an odd-sized window on the image carrier, and then to replace the central pixel with the median value of the pixels.

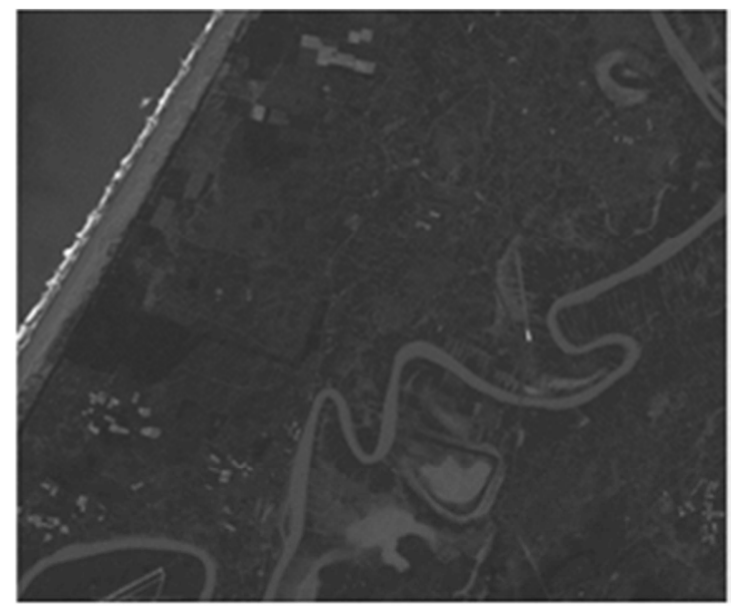

(a)

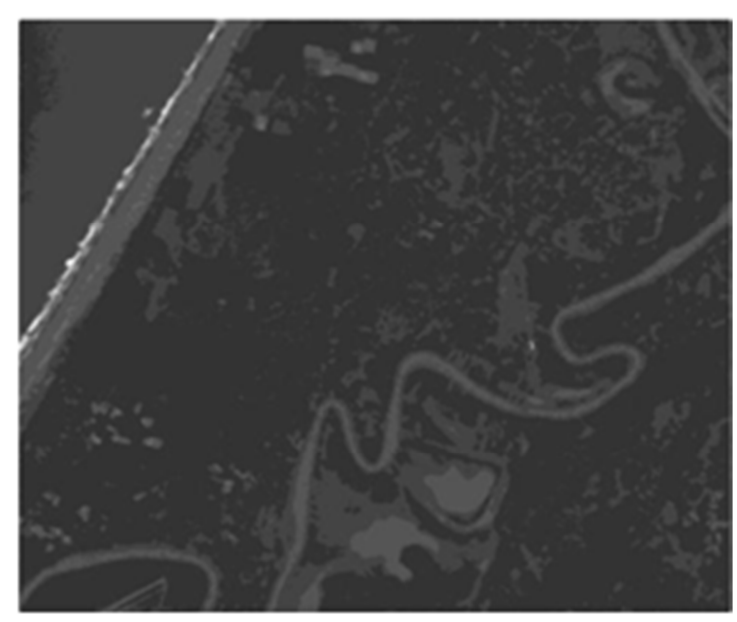

(b)

Figure 3. Band $n 1$ of the reference image (Kenitra region)(a-Pre-processing. $\boldsymbol{b}$ - Post-processing using $3 * 3$ window). 


\section{Non-Supervised Classification}

To extract the spectral signatures [5] of different targets on the reference image, we proceeded firstly to a non-supervised classification and the confrontation of the chosen classes with those of the reference field.

To do this, we used a connectionist method based on Kohonen Self Organizing Map (SOM) [6,7]. This method allowed to make an effective classification despite the influence of the physical parameters, including the atmosphere, the sensor configuration and topography. These factors seriously affect the quality of the classification in the case of conventional approaches (maximum likelihood, ISODATA, etc.) [8].

SOM ANNs maps are mainly used for clustering and non-linear projection of the data $[9,10]$.

\subsection{Network Architecture}

The network built from classifying spectral data of the image studied is a two-dimensional self-organizing map consisted of nine neurons $(3 * 3)$ (Figure .3 ).

The choice of this dimension is due to the fact that nine classes are sufficient and effective to distinguish four targets. In effect, a larger dimension gives more classes and makes therefore the distinction between classes very difficult on terrain. Also, a smaller size will not provide enough of classes among which we can choose our targets.

The input vector $E=$ [e1; e2; e3; e4; e5; e6; e7] represents the standard reflectance of the seven bands characterizing a voxel, and is linked to all neurons. $\quad\left(\mathrm{e}_{i}\right.$ is the vector of $393 * 473$ pixels of the band $i$ ).

External connections of the neuron $i$ with the input vector $\mathrm{E}$ are materialized by a synaptic weight vector $P i=\left[\mathrm{p}_{\mathrm{i} 1} \mathrm{p}_{\mathrm{i} 2} \ldots \mathrm{p}_{\mathrm{i}}\right]$, with $i=1, \ldots, 9$.

Similarly, neurons of the map are interconnected (internal connections) and their connection weights $W_{i j}$ are expressed by a Gaussian "Mexican hat" [11]. The feature of these interactions is that they depend on the distance between the neurons.

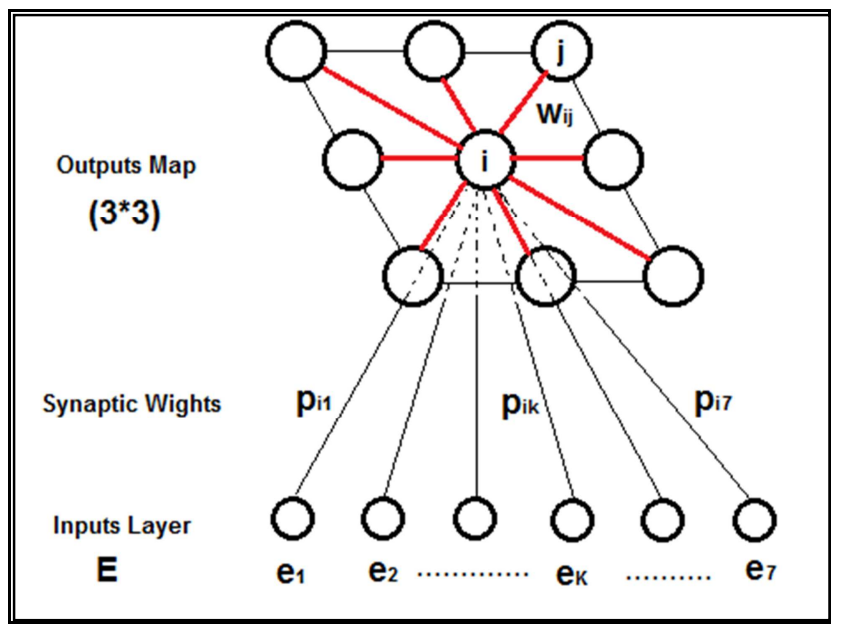

Figure 4. Architecture of the proposed $\operatorname{SOM}(3 * 3)$.
The activation function of the network used for the evaluation of the output is a sigmoid $f$ :

$$
f(x)=\beta\left(\frac{e^{\alpha x}}{\left(1+e^{\alpha x}\right)}\right)
$$

Since the manipulated data is normalized, we take and we shall have:

$$
f(x)=\frac{e^{x}}{1+e^{x}}
$$

The output $S_{i}$ of the neuron $i$ at time $t$ is then expressed as follows:

$$
S_{i}(t)=f\left[\sum_{k} p_{i k} \cdot E_{k}+\sum_{\substack{j \\(i \neq j)}} w_{i j} S_{j}(t-1)\right]
$$

It is based on the weighted sum of the inputs $\sum p_{\mathrm{ik}} . \mathrm{E}_{\mathrm{k}}$ and the weighted sum of the outputs of neighboring neurons at time t: $\sum w_{i j} \cdot S_{j}(t-1)$.

\subsection{Learning Process of the SOM}
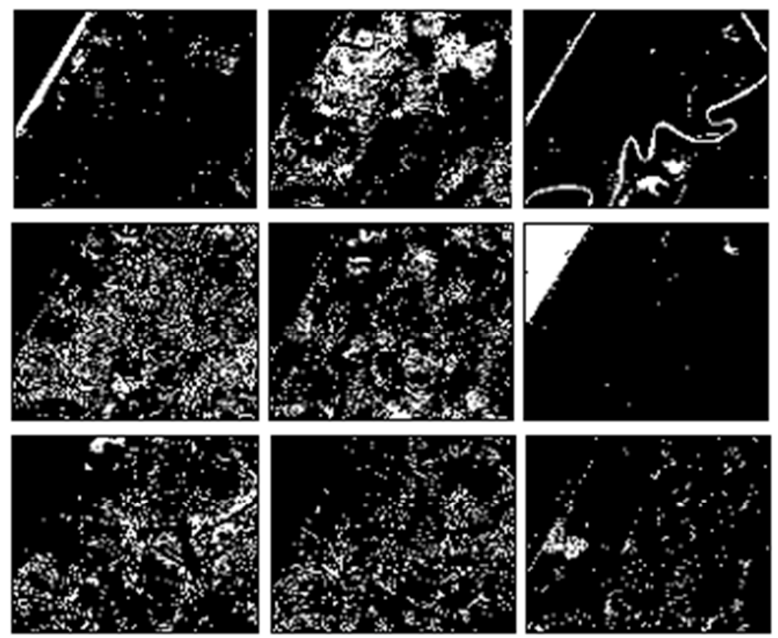

Figure 5. Images of the nine resulting classes (from SOM).

During the training phase, the network adapts to different data bands. This step is basically to change the weight of the external connections between neurons of the map and the inputs and to strive for optimum adaptation of the network. Learning process takes place according to the following rule [9]:

- Initialization of the side links $P_{i}$ (weight vectors) to some small random variables;

- Presentation of the input vector E;

- Calculate the Euclidean distance between the input vector $E$ and $P_{i}$ vectors for each neuron of the map and select the nearest neuron $j$ to $E$ : 


$$
\begin{gathered}
\left\|E-P_{i}\right\|=\sqrt{\sum_{j}\left(E_{j}-P_{i j}\right)^{2}} \\
d_{j}=\left\|E-P_{c}\right\|=\min \left(\left\|E-P_{i}\right\|\right)
\end{gathered}
$$

2. Learning and update of the weights $P_{k}$ according to the neighborhood rule $h\left(k, d_{j}\right)$ :

$$
P_{k}(t+1)=P_{k}(t)+\rho(t) h\left(k, d_{j}\right) \cdot\left(E(t)-P_{k}(t)\right)
$$

$h$ is the neighborhood rule and $\rho$ the learning step value. Repeat steps 2, 3 and 4 until the complete stabilization of the weights. The neighborhood is shrinking and the learning coefficient decreases over time. Thus, at the end of the learning process, the map is self-organized into nine groupings. After resizing the nine outputs and the establishment of new matrices (393x473), we obtain new binary images, each representing a different class (Fig. 5).

\subsection{Building of the Database}

At this stage, we compared the binary images obtained with real aspect of the ground. This allowed us to identify the areas of the desired target on the original image (Fig. 6).
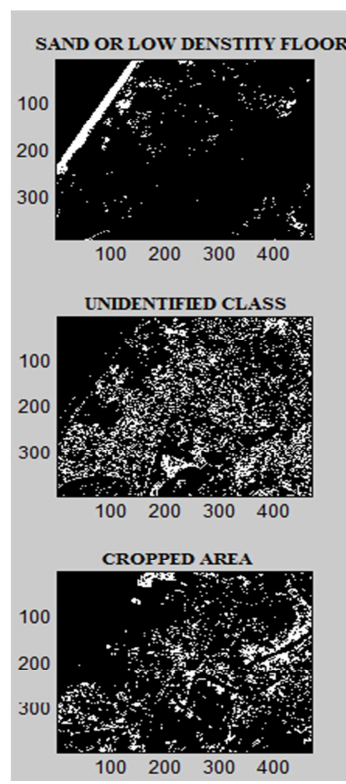
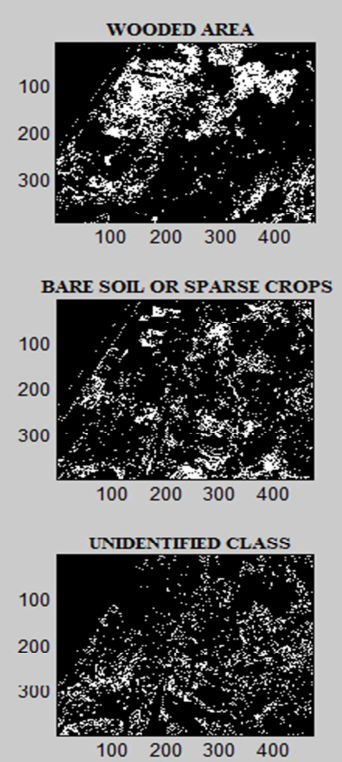
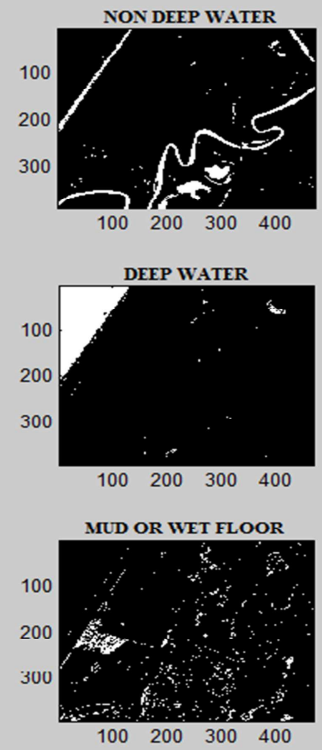

Figure 6. Classification of the SOM images.

This comparison is simplified by the color composite images containing the desired classes (Fig.7).

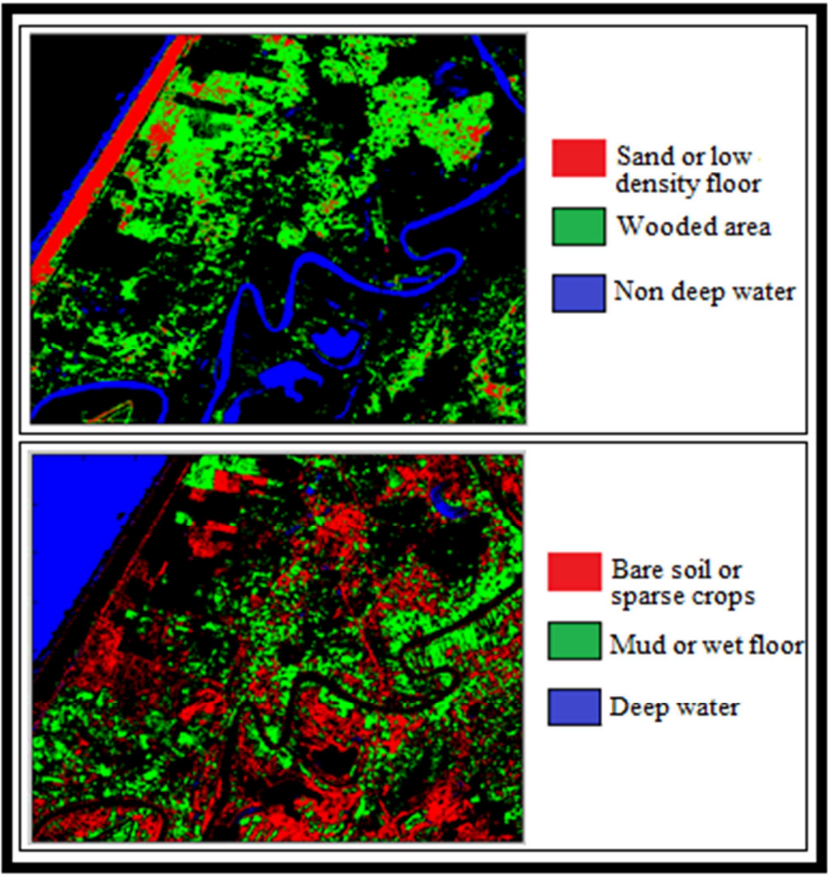

Figure 7. Classification of the desired target on the basis of colored compositions of the images obtained by the SOM map. 
Finally, once the targets are identified on the seven reference bands image, we extracted eight samples of the normalized reflectances of the aforesaid each target.
In this study, we chose the most relevant and essential: non deep water, sandy soil, wooded area and wet soil (Table 1).

Table 1. Samples of the targets taken from the (7-bands) reference image.

\begin{tabular}{|c|c|c|c|c|c|c|c|c|}
\hline \multirow{2}{*}{$\begin{array}{l}\text { Bands } \\
1\end{array}$} & \multicolumn{8}{|c|}{ SAND OR LOW DENSITY FLOOR } \\
\hline & 0,21 & 0,21 & 0,21 & 0,21 & 0,20 & 0,21 & 0,21 & 0,21 \\
\hline 2 & 0,33 & 0,32 & 0,32 & 0,32 & 0,35 & 0,34 & 0,34 & 0,32 \\
\hline 3 & 0,35 & 0,34 & 0,34 & 0,34 & 0,36 & 0,4 & 0,33 & 0,41 \\
\hline 4 & 0,33 & 0,33 & 0,32 & 0,33 & 0,34 & 0,33 & 0,33 & 0,32 \\
\hline 5 & 0,28 & 0,29 & 0,26 & 0,3 & 0,31 & 0,32 & 0,27 & 0,29 \\
\hline 6 & 0,52 & 0,52 & 0,52 & 0,52 & 0,53 & 0,52 & 0,52 & 0,52 \\
\hline \multirow[t]{2}{*}{7} & 0,27 & 0,21 & 0,29 & 0,21 & 0,31 & 0,36 & 0,29 & 0,3 \\
\hline & \multicolumn{8}{|c|}{ NON DEEP WATER } \\
\hline 1 & 0,61 & 0,61 & 0,61 & 0,61 & 0,62 & 0,62 & 0,69 & 0,68 \\
\hline 2 & 0,72 & 0,68 & 0,67 & 0,67 & 0,67 & 0,67 & 0,69 & 0,66 \\
\hline 3 & 0,55 & 0,64 & 0,53 & 0,54 & 0,54 & 0,55 & 0,54 & 0,53 \\
\hline 4 & 0,45 & 0,54 & 0,53 & 0,54 & 0,54 & 0,54 & 0,55 & 0,54 \\
\hline 5 & 0,46 & 0,45 & 0,45 & 0,45 & 0,45 & 0,45 & 0,45 & 0,44 \\
\hline 6 & 0,42 & 0,42 & 0,42 & 0,42 & 0,42 & 0,42 & 0,42 & 0,42 \\
\hline 7 & 0,54 & 0,43 & 0,47 & 0,57 & 0,64 & 0,75 & 0,4 & 0,6 \\
\hline 2 & 0,61 & 0,59 & 0,58 & 0,56 & 0,61 & 0,59 & 0,57 & 0,56 \\
\hline 3 & 0,34 & 0,34 & 0,33 & 0,33 & 0,34 & 0,34 & 0,34 & 0,33 \\
\hline 4 & 0,34 & 0,34 & 0,33 & 0,33 & 0,34 & 0,35 & 0,34 & 0,34 \\
\hline 5 & 0,65 & 0,64 & 0,64 & 0,64 & 0,65 & 0,64 & 0,64 & 0,64 \\
\hline 6 & 0,02 & 0,01 & 0 & 0,01 & 0,02 & 0,02 & 0 & 0 \\
\hline \multirow[t]{2}{*}{7} & 0,41 & 0,39 & 0,38 & 0,36 & 0,41 & 0,38 & 0,37 & 0,34 \\
\hline & \multicolumn{8}{|c|}{ WOODED AREA } \\
\hline 1 & 0,31 & 0,31 & 0,31 & 0,31 & 0,31 & 0,31 & 0,31 & 0,31 \\
\hline 2 & 0,41 & 0,39 & 0,38 & 0,36 & 0,31 & 0,39 & 0,34 & 0,36 \\
\hline 3 & 0,34 & 0,34 & 0,33 & 0,34 & 0,34 & 0,33 & 0,33 & 0,34 \\
\hline 4 & 0,34 & 0,34 & 0,43 & 0,33 & 0,34 & 0,35 & 0,34 & 0,04 \\
\hline 5 & 0,01 & 0,01 & 0,01 & 0,01 & 0 & 0 & 0 & 0,01 \\
\hline 6 & 0,05 & 0,04 & 0,01 & 0 & 0,01 & 0 & 0 & 0 \\
\hline 7 & 0,01 & 0 & 0 & 0 & 0,01 & 0 & 0 & 0 \\
\hline
\end{tabular}

We see that the reflectance values obtained in table 1 differ from one band to another and from one class to another. This indicates and confirms the difference of the answer of the target classes to the wavelengths emitted by the onboard radar (spectral signatures). In addition, for samples of the same strip, these values are very close together, and even equal in some cases, which shows the effectiveness of the made classification.

\section{Conclusions}

In this paper, we proceeded to the application of a nonlinear median filter type over a multiband image. This filtering enabled us to eliminate the speckle noise and preserve the shape of the boundaries between the different classes of the image. Then we presented a connectionist approach to building a database on four targets of major interest. To do this, we performed a clustering filtered images representing the seven bands and, using a map scale SOM $\left(3^{*} 3\right)$. This network has allowed us to define new classes on this image. Comparison of these classes with the real aspects of the ground finally allowed us to define the nature and position of our target on the satellite image and obtain therefore a reference reflectance database.

The target identification studied on other images taken by the same sensor can be performed thereafter with a supervised classification of the reference data.

Thus, thanks to the robustness of their models, Artificial Neural Networks seem to be remarkably well adapted to different targets. Their adaptation to nonlinear problems, is a major asset for a very *satisfactory solution to the classification and identification.

\section{References}

[1] Mukesh C. Motwani, Mukesh C. Gadiya, Rakhi C. Motwani, Frederick C. Harris, Jr, "Survey of Image Denoising Techniques," Proc. of GSPx 2004, Santa Clara Convention Center, Santa Clara, CA, 2004, pp. 27-30.

[2] H. Guo, J. E. Odegard, M. Lang, R. A. Gopinath, I.W. Selesnick, and C. S. Burrus, "Wavelet based Speckle Reduction with Application to SAR based ATD/R," First International Conference on Image Processing, 1, 1994, pp. 75-79.

[3] Maïtine Bergounioux. Quelques méthodes de filtrages en traitement d'image.CIMPA, hal-00512280v1, 2011. 
[4] E. Nezry, Amélioration radiométrique et préservation de la réflectivité radar des images SAR par les filtres de speckle adaptatifs : Théorie et résultats expérimentaux, Centre commun de recherche, Institut des applications de la télédétection, Commission européenne, 1994, pp.3-7.

[5] J. Philippe, G. Etchegorry, F. Adragna, Y. Kerr, J. Lagouarde, M. Leroy et $\mathrm{T}$. Le Toan, Observation spaciale des paramètres de surface, (Paramètres de surface et signatures spectrales), La télédétection en Francophonie : analyse et perspectives, Edition AUF2000, pp. 299-317.

[6] N. Allinson, H. Yin, N. Allinson and J. Slack Eds. Self-Organizing Maps.Springer, 2001, pp.95-101.

[7] T. Kohonen, Essentials of the self-organizing map, Neural Networks 37, 2013, pp. 52-65.
[8] T. Kohonen, Self-Organizing Maps, Springer Series in Information Sciences, Vol.30, $2^{\circ}$ edition, 1997.

[9] M. Hendel, A. Benyettou, F. Hendel, H. Khilil, « Classification automatique des signaux ECG par les réseaux de neurones probabilistes ", "Applications Médicales de l'Informatique : Nouvelles Approches", Monastir-Tunisie, 13, 14 et 15 Novembre 2008.

[10] R. Le page, Détection et analyse de l'onde $\mathrm{P}$ d'un électrocardiogramme : application au dépistage de la fibrillation auriculaire, Thèse Doctorat d'état électronique. L'université de Bretagne occidentale, 2003.

[11] Teuvo Kohonen, Jussi Hynninen, Jari Kangas and Jorma Laaksonen. Som-pak, the self-organizing map program package. SOM programming team of Helsinki University of Technology, April 1995. 